\title{
Die deutschsprachige Lyrik und die ,Stunde Null' 1945
}

\section{German Poetry and the 'Stunde Null' 1945}

\author{
Jana Hrdličková
}

\begin{abstract}
If one attempts to establish periods of the development of German poetry in the 20th century, the year 1945 proves to be rather an ambivalent date. On the one hand, the political upheaval clearly had its effects on poets; on the other hand, a strong continuity can be observed in the style and subject-matter of many works written in the 1930s and the 1940s or even later.

The aim of this paper is to show whether and how the year 1945 influenced German-language poetry by examining texts written by authors as different as Nazi-sympathizer Hans Egon Holthusen, resistance fighter Albrecht Haushofer, authors of the so-called 'Innere Emigration' - Gottfried Benn, Marie Luise Kaschnitz and Günter Eich - and by taking into account the famous poem about the Shoa, Death Fuge by Paul Celan, who emigrated.
\end{abstract}

\section{Keywords}

caesura constructiones in literature; German poetry from the 1930s to the 1950s; the 'Stunde Null' 1945, Hans Egon Holthusen; Gottfried Benn; Günter Eich; Marie Luise Kaschnitz; Paul Celan 
Als Hermann Korte 1999 die deutschsprachige Lyrik des 20. Jahrhunderts auf ihre Zäsuren hin untersuchte, wollte er den „müßige[n] Streit um sozialgeschichtliche oder innerliterarische Zäsur-Konstruktionen" (Korte 1999: 63) bewusst umgehen. Denn dieser Streit sei, wie er betonte, „inzwischen ein Teil der Wissenschaftsgeschichte geworden“ (Korte 1999: 63), also kaum zeitgemäß. Er verstand „gewisse Trenndaten“ zur „Markierung von Einschnitten in literarhistorischen Prozessen“ als nützlich, wenn auch jeweils „von der Strategie abhängig, mit der Geschichten über Literatur erzählt werden“. (Korte 1999: 63)

Daher wollte er selbst auch nicht „die Lyrikgeschichte des Säkulums“ liefern, sondern „nur eine ihrer vielen Geschichten“. Diese seine Geschichte bestimmte er im nächsten Schritt als „die Geschichte der lyrischen Moderne von 1890 bis heute“. (Korte 1999: 63)

Interessant ist, dass seine Periodisierung der lyrischen Entwicklung des 20. Jahrhunderts dann viele innerliterarische Trenndaten kennt, in der Reihenfolge 1890/1900 (mit George, Rilke und Hofmannsthal als Gewährsmännern der Abgrenzung zur Erlebnisund Stimmungslyrik des späten 19. Jahrhunderts), 1910 (als die Expressionisten die Avantgarde der Jugend begründeten), 1920 (mit der Kabarettlyrik und der Dada-Ära), 1930 (mit poetologischer, Verlangsamungsstrategie، und ihrem Ausdruck in der Naturlyrik Lehmanns und Loerkes), und schließlich 1960 (verbunden mit der Konkreten Poesie und dem Aufstieg ,zorniger junger Männer‘ wie Enzensberger; vgl. Korte 1999: 88).

Nur drei Bruchstellen sind bei ihm geschichtlicher oder politischer Natur. Und sie sind sogar darüber hinaus jedes Mal in der Überschrift mit drei Punkten eingeleitet sowie am Ende mit einem Fragezeichen versehen, also in ihrer Gültigkeit und Aussagekraft relativiert: „... und 1933?“ (Korte 1999: 81), „... und 1945?““ (Korte 1999: 85) sowie „... und 1990?“ (Korte 1999: 95). So wird bei der Zäsur des Jahres 1933 zwar eingeräumt, dass sie ,in die Lebensgeschichte vieler Lyrikerinnen und Lyriker [markant] eingriff“" (Korte 1999: 82), was natürlich auch ihre dichterische Praxis prägte, poetologisch gesehen jedoch nur „die traditionalistische Bruchstelle von 1930 verstärkte und noch sichtbarer machte“. (Korte 1999: 82) Denn, so Korte (1999: 81): „Die Option für traditionalistische Schreibtechniken war keine Weltanschauungsfrage“. Die Form des Sonetts beispielsweise florierte weit über das Jahr 1945 hinaus „bei den Exildichtern ebenso wie bei jenen der ,Inneren Emigration““ (Korte 1999: 83), „im Gestapogefängnis, im Konzentrationslager, in Kriegsgefangenschaft und in der Kammer des Einzelnen, der sich mindestens geistig dem Regime entgegenstellen“ wollte, wie Theodor Ziolkowski (1972: 156) weiter exemplifiziert.

Sogar die Mitläufer des NS-Regimes, die weder Korte noch Ziolkowski bei ihrer Aufzählung erwähnen, suchten in der Form des Sonetts „Einsicht, Halt, Trost, verbindliche Weltschau, wahre Botschaft und sicheres Fundament zugleich“. (Korte 1999: 84) So versucht Hans Egon Holthusen, der bereits 1933 freiwillig in die SS-Standarte Julius Schreck eintrat $^{1}$ und 1940, mit 27 Jahren, in der ,gleichgeschalteten` evangelischen Zeitschrift

$1 \quad$ Er soll sich noch 1944 in SS-Uniform gezeigt haben, obwohl er nach eigenen Angaben nur bis 1937 aktives Mitglied war (vgl. Rduch 2012: 148) und sich selbst sogar „[g]egen Ende des Krieges [als] Mitwisser und Parteigänger einer militärischen Verschwörung gegen das Naziregime“ bezeichnete. (Holthusen 1966a: 923) Dass „eine[] bekannte[] amerikanische[] Zeitschrift [ihn 1946] als Dichter des deutschen Widerstandes genannt“ 
Eckart den Überfall auf Polen mit dem „ein Jahrtausend alt[en]“ Spruch der „niederdeutschen Ordensritter und Siedler“, nämlich „Nach Osten wollen wir reiten““, rechtfertigte (Holthusen 1940: 304), seine eigenen Erfahrungen an der Ostfront und den Verlust des Bruders ,im Osten“ in die folgende Totenklage $(1943)^{2} \mathrm{zu}$ fassen:

„Es war mein Blut, das sich aus dir ergossen.

Nun ist mir alles fremd. Nun gehst du tot

In dieses Dasein ein. So sei mein Brot

Hinfort nicht ohne deinen Tod genossen.

Mein Herz fühlt aus den Angeln sich gehoben

Und sieht die Dinge schwindlig und verändert.

Die Blumen sind mit Todeslicht gerändert,

Und alle Horizonte sind verschoben.

O furchtbar, einen Bruder zu besitzen

Und einmal nur - dies Kreuz ward uns gesetzt -

Berühren dürfen mit den Fingerspitzen!

Ein kleiner Ruck der Zeit hat dich getroffen,

Ein bloßer Lidschlag zwischen jetzt und jetzt,

Und meine Arme bleiben ewig offen.“ (Holthusen 1979: 358)

Dieses Gedicht, das schon während des Kriegs geschrieben und erstmals veröffentlicht wurde, allerdings erst nach seinem Ende bekannter wurde, kann bereits die „ungewöhnliche Windstille“ und „nachsintflutliche Atmosphäre“ illustrieren, die Viktor Žmegač den Dichtern in ihrer Antwort auf die Niederlage Deutschlands im Zweiten Weltkrieg attestierte, im Kontrast zu dem Zustand nach dem Ersten Weltkrieg, als sich die „expressionistische Aufbruchsstimmung“ ausbreitete. (Žmegač 1981: 374)

Darüber hinaus versinnbildlicht Holthusens Text einprägsam Kortes „... und 1945?“ benannte - oder eben in Frage gestellte - Zäsur, die uns hier besonders interessiert. Denn diese bemüht regelmäßigen Jamben und der wenig einfallsreiche, eher durchschnittliche und fast ermüdende Reim („ergossen“/,genossen“, „verändert“/,gerändert“)

habe, erwähnt er im nächsten Schritt. (Holthusen 1966a: 923) Dass diese Charakterisierung nicht der Wirklichkeit entspricht, beweist Robert Rduch durch eine Analyse von Holthusens Beträgen für die Zeitschrift Eckart. (vgl. Rduch 2012: 147-157, v.a. 149-151)

2 Das Sonett ist erstmals 1943 als erster Text des Sonettzyklus Eine Totenklage. In memoriam Walter Holthusen, gefallen am 18. August 1942, im Osten in der erwähnten Zeitschrift Eckart erschienen, neben acht anderen Sonetten. Nach dem Krieg publizierte es Holthusen in der Zeitschrift Die Wandlung neben sieben Sonetten im Jahre 1946, in der Broschüre der Reihe Das Gedicht - Blätter für die Dichtung unter dem Titel Klage um den Bruder 1947 als zweites von 14 Sonetten, schließlich in dem Gedichtband Hier in der Zeit (1949) neben elf Sonetten. (vgl. auch Rduch 2012: 151-152) Diese Frequenz lässt erahnen, wie wichtig der Text dem Autor war, zugleich aber bekommt er durch seine Wiederholungen einen fast kanonischen Charakter, zumindest im Oeuvre Holthusens. Die Wichtigkeit des Einschnitts von 1945 wird durch diesen kontinuierlichen Einsatz so gut wie geleugnet. 
weisen auf „die weithin ungebrochene Kraft des Traditionalismus in allen seinen Facetten [hin sowie auf die Tatsache], daß er mühelos über die einschneidendste politische Zäsur des Jahrhunderts hinwegkam und sich behauptete.“ (Korte 1999: 86)

Dies zeigt sich auch auf der thematischen Ebene. Der im Krieg Gefallene, dessen Blut in einer auffälligen Nähe zu den Worten Jesu während des letzten Abendmahls („Das ist mein Blut, das für alle Menschen vergossen wird“, Mk 14, 24) zum Blut des lyrischen Ichs, des Bruders, erklärt wird - allerdings im unwiederbringlichen Präteritum („Es war mein Blut, das sich aus dir ergossen“, Hervorhebung J.H.) - wird im Gedicht voller Melancholie und Trauer angesprochen, sein gewaltsamer Tod im Krieg jedoch zugleich verschleiert, oder vielmehr ganz ausgespart, und als etwas Notwendiges, durch die christliche Überlieferung Verbürgtes (s. die erneute biblische Allusion in Zeile 10: „dies Kreuz ward uns gesetzt") und Zeittypisches dargelegt; heißt ja die Sammlung, in die das Gedicht schließlich, 1949, aufgenommen wurde, wie erwähnt Hier in der Zeit.

Diese Taktik der Meidung des konkreten Todesumstands im Krieg bemerkt auch Robert Rduch in seiner Interpretation des Gedichts. Er unterstreicht, dass „der Außenwelt lediglich in der letzten Strophe Aufmerksamkeit geschenkt wird“, wobei „der Krieg als Ursache des Todes, jedweder Konkreta beraubt, zu einem abstrakten Geschichtsphänomen“ wird. (Rduch 2012: 152) Vor allem die Zeilen 12 und 13, „Ein kleiner Ruck der Zeit hat dich getroffen,/ Ein bloßer Lidschlag zwischen jetzt und jetzt“, begeben sich in den Schutz des völlig Abstrakten, seltsam Metapherhaften („Ruck der Zeit“ und „Lidschlag“" als Agenzien der Vernichtung) und dem Kriegsgeschehen Abgewandten, geben alle Schuld am Tod des Bruders - „der Zeit“. Holthusens eigenes Engagement für das NS-Regime und seine Begeisterung für den Ritt „Nach Osten“ werden dadurch tabuisiert und im Unverbindlichen aufgehoben. Dem lyrischen Ich wird zwar „alles fremd“ (Zeile 2), sein „Brot“ schmeckt ihm nach Tod (Zeile 3 und 4), sein Herz gerät „aus den Angeln“ (Zeile 5), es sieht „die Dinge schwindlig und verändert" (Zeile 6), was alles eine Chance zum Sprung auf eine neue Erkenntnisstufe, die den ,verschobenen Horizonten (Zeile 8) gerecht wäre, bilden könnte... den jedoch das lyrische Subjekt schließlich doch nicht wagt. Stattdessen wird bei der christlichen Überlieferung, zudem im archaischen Ton (vgl. nochmals die Mitte der vorletzten Strophe: „dies Kreuz ward uns gesetzt“, wobei das antiquierte „ward“ hervorsticht), Zuflucht gefunden und ganz zuletzt im schwer Verständlichen, Schleierhaften (Zeile 9-11: man besitzt einen Bruder, darf - ihn? nach seinem Tod? - aber nur einmal berühren „mit den Fingerspitzen!“). Dass diese Lyrik „,in der Nachfolge Rilkes [stehe], [...] formal aber strenger [ist]“ (Kircher 1979: 491), lässt sich anhand dieses Beispiels, das Hartmut Kircher immerhin für seine Anthologie Deutsche Sonette als einzig repräsentativ für Holthusens Schaffen auswählte, wahrhaftig nicht sagen.

Das Genre des Sonetts kann bei aller Achtung der Form aber auch völlig anders gehandhabt werden, wie es der Text von Albrecht Haushofer zeigt, eines Autors, der als Geograf Rudolf Heß und Joachim von Ribbentrop beriet und als Experte für internationale Beziehungen des NS-Staates galt, der jedoch seit Frühjahr 1940 (als der zehn Jahre jüngere Holthusen noch kriegsbegeistert war) als Widerstandskämpfer figuriert. Nach dem gescheiterten Attentat auf Hitler vom 20. Juli 1944, das er persönlich ablehnte, 
wurde er verhaftet und ins Gefängnis Berlin-Moabit gebracht. (vgl. Sarkowicz - Mentzer 2000: 188) Nur wenige Tage vor der Befreiung Berlins durch sowjetische Truppen, in der Nacht vom 23. auf den 24. April 1945, wurde er zusammen mit fünfzehn anderen Häftlingen von der SS hinterrücks erschossen. (vgl. Sarkowicz - Mentzer 2000: 189) In seiner Manteltasche fand sein Bruder 79 Gedichte (vgl. Sarkowicz - Mentzer 2000: 189), unter anderem das folgende Sonett (das Hartmut Kircher in seiner Anthologie Deutsche Sonette direkt nach Holthusens Es war mein Blut anordnet):

\begin{abstract}
„Schuld
Ich trage leicht an dem, was das Gericht mir Schuld benennen wird: an Plan und Sorgen. Verbrecher wär' ich, hätt' ich für das Morgen des Volkes nicht geplant aus eigner Pflicht.

Doch schuldig bin ich anders als ihr denkt, ich mußte früher meine Pflicht erkennen, ich mußte schärfer Unheil Unheil nennen mein Urteil hab ich viel zu lang gelenkt...
\end{abstract}

Ich klage mich in meinem Herzen an:

Ich habe mein Gewissen lang betrogen, ich hab mich selbst und andere belogen -

ich kannte früh des Jammers ganze Bahn ich hab gewarnt - nicht hart genug und klar!

Und heute weiß ich, was ich schuldig war..." (Haushofer 1979: 359)

Hier kann von einem langweiligen formalen Aufbau schon deshalb keine Rede sein, weil der Text zwar streng die Gesetze des Sonettbaus befolgt (Reimschema abba abba cdd - nur in der letzten Strophe figuriert statt cee das markantere, schärfere ccc; Wechsel von männlichem und weiblichem Schluss; durchgehende jambische Fünfheber), trotzdem aber über einen originellen, mannigfaltigen und dynamischen Redefluss verfügt, den nicht zuletzt die satzabschließenden Zeichen (Punkt, Komma, Gedankenstrich, Doppelpunkt, Ausrufezeichen, drei Punkte) modellieren sowie die zahlreichen, Ich-zentrierten Parallelismen („ich mußte früher meine Pflicht erkennen,/ ich mußte schärfer Unheil Unheil nennen - " usw.).

Noch mehr unterscheidet sich dieses Sonett vom Text Holthusens unter inhaltlichem Gesichtspunkt. Denn obwohl auch Haushofer vom christlich-humanistischen Weltbild geprägt wurde (vgl. Sarkowicz - Mentzer 2000: 189), gibt es in seinem Sonett kein Versteckspiel mit dem Krieg, der bei Holthusen nur höchst euphemistisch, durch eine Personifikation, angegangen werden konnte (vgl. die schon zitierte Zeile 12: „Ein kleiner Ruck der Zeit hat dich getroffen“), und auch keine Rückendeckung durch das vermeintlich ewige und einzig wahre Christliche. 
Vielmehr hält das lyrische Subjekt in Haushofers Sonett eine Art Gericht über sich selbst, mit eindeutigen, scharfen, präzise argumentierenden Worten, denen es um Erkenntnis und vielleicht auch um Buße geht. Sarkowicz und Mentzer (2000: 189) zufolge stellen sie „ein in der jüngeren deutschen Literatur fast einmaliges Bekenntnis zur eigenen Schuld" dar, wobei jede der insgesamt neun staccatoartigen Zeilen, die ab Vers 6 das Personalpronomen „Ich“ in der Spitzenstellung unter Beschuss nehmen und es im Zusammenhang mit der privaten Beichte hervorheben, zugleich weit entfernt vom Sprechen in nur eigener Sache, das lyrische Aussagen per se häufig prägt, ist. Diese Sätze sollen angesichts des antizipierten, nahen Todes an das Mitgefühl des Lesers appelieren, wollen offenbar, um mit Kafka zu sprechen, „die Axt sein für das gefrorene Meer in uns“ (Kafka 1998: 29). Durch diese Intention wird Haushofers subjektives Empfinden objektiviert, gelangt zu jener „gültigen Aussage“ (Arndt 1989: 200), die Erwin Arndt von einem Sonett fordert. Holthusens Sonett dagegen, das im subjektiven Schmerz um den Verlust des Bruders aufgeht, ohne den Mut zu Fragen nach Voraussetzungen dieses Todes und Schlussfolgerungen daraus zu ziehen, verfehlt offensichtlich eine solche Zielsetzung.

1966 noch, im gleichen Jahr, als Jean Améry in seinem Buch Jenseits von Schuld und Sühne. Bewältigungsversuche eines Überwältigten mit erhellenden wie schockierenden Passagen über die eigene SS-Tortur kommt, findet Holthusen in seinem als Rechtfertigung konzipierten Aufsatz Freiwillig zur SS, in seiner Stammzeitschrift Merkur veröffentlicht, keinen Mut zur Reue, auch keinen Mut zum eigenen Ich. Er spaltet hier nämlich sein gegenwärtiges, anerkanntes Alter Ego (,christlicher Existentialist“, „inmitten der literarischen Welt Deutschlands“ Stehender", seit 1959 als Gastprofessor an verschiedenen Universitäten der USA, 1961-64 zudem als Programmdirektor am Goethe-Haus in New York tätig) von gleich mehreren knabenhaften Personen, die er behaglich als „Monsieur“ (Holthusen 1966a: 925), „[m]ein Tagebuchschreiber“ (Holthusen 1966a: 926), „mein Tonio Kröger“ (Holthusen 1966a: 928), „mein Milchbart“ (Holthusen 1966a: 928) sowie „[m]ein Mandant“ (Holthusen 1966a: 928), „unser[] Pastorensohn“ (Holthusen 1966a: 929) oder „unser Hans Dampf“ (Holthusen 1966a: 931) einführt und väterlich verteidigt.

Das dies die Integrität der eigenen Person nicht wiederherstellen kann, liegt auf der Hand, zudem beispielsweise Holthusens NSDAP-Zugehörigkeit seit dem Jahr 1937 völlig ausgeblendet wird. In einer Fortsetzung dieses Unterfangens, einen Monat später im Merkur publiziert, empfindet es sein „Mandant“ schließlich als „Zumutung, [...] von weiß wem einer verhörartigen Befragung unterworfen zu werden“ (Holthusen 1966b: 1043), und nennt in Churchill, Daladier, Papst Pius und anderen aussichtsreichere Anwärter auf die Bezeichnung „ein Nazi“ (Holthusen 1966b: 1046-1047). An Benn geschult, den er nicht zuletzt im Essay Die Überwindung des Nullpunkts seines ikonischen Buchs Der unbehauste Mensch (1951) umfassend würdigt, plädiert er für „die Welt des Einzelnen in ihrer kategorischen Bestimmtheit durch die Schicksalsmächte Liebe und Tod, Glück und Unglück, Jugend und Alter, das Geheimnis, das wir Gott nennen, und das Rätsel, das wir uns selber sind, aber auch in ihrer praktisch-nothaften Angewiesenheit auf Arbeit, Lebenskampf, Fürsorge für andere usw. [...]“. (Holthusen 1966b: 1041) In dem

3 So auch von Jean Améry (2005: 47) tituliert und eingeordnet. 
Sinne antwortet er auch Hannah Arendt auf ihr „berühmtes Wort von der ,organisierten Schuld““ (Holthusen 1966b: 1041): Er leugnet es, „daß es die politische Sphäre ist, in der der Mensch als Person, der Mensch in seiner Gänze, mit allen seinen Wesensbereichen seine Rechtfertigung empfängt." (Holthusen 1966b: 1042) Vor diesem für ihn unwesentlichen Politischen sucht er Zuflucht im Privaten.

Dies ähnelt zwar tatsächlich, wenn auch nur zum Teil, Gottfried Benn, der schon seit seiner Abkehr vom Expressionismus in den Zwanzigerjahren Korte zufolge „keine weltanschaulichen Botschaften im Gedicht, keine Trost- und Warnfunktion, keine lyrische Prophetie und kein moralisches Amt“ (Korte 1999: 85) anerkenne. Aber ist diese weitverbreitete Meinung, die Benn selbst durch seine Aussagen und Briefe wohl stützen mochte, tatsächlich pauschal gültig?

Denn Benns letzte Buchpublikation während des ,Dritten Reichs', der Band Ausgewählte Gedichte, im Mai 1936 zu seinem 50. Geburtstag veröffentlicht mit Texten aus den Jahren 1911 bis 1936, scheint eine andere Sprache zu sprechen und eine Entwicklung anzudeuten, an deren Endstufe erst das berühmte apolitische, kosmisch „[v]erlorene[] Ich“ aus den Statischen Gedichten (1948) der Nachkriegszeit steht - wobei das Gedicht Verlorenes Ich allerdings schon 1943 erstmals veröffentlicht wurde.

In diesen Ausgewählte[n] Gedichten, die nach Hillebrand ausgezeichnete Kritiken ernteten (vgl. Hillebrand 1992: 602), vom Redakteur der SS-Zeitung Das schwarze Korps allerdings unter dem Titel Der Selbsterreger! attackiert wurden, da sie „Ferkeleien“, „widernatürliche Schweinereien“ und „Geistesverblödung“ seien (vgl. Sarkowicz - Mentzer 2000: 84), ${ }^{4}$ findet sich ein Gedicht, das sehr im Kontrast zu diesen wüsten Beschimpfungen erscheint - Anemone. Kortes Worte über die fehlenden weltanschaulichen Botschaften bei Benn, über die Absenz von Trost- und Warnfunktion in seinem Schaffen gelten hier m. E. nicht:

„Anemone
Erschütterer - : Anemone,
die Erde ist kalt, ist Nichts,
da murmelt deine Krone
ein Wort des Glaubens, des Lichts.

Der Erde ohne Güte,

der nur die Macht gerät,

ward deine leise Blüte

so schweigend hingesät.

Erschütterer - : Anemone,

du trägst den Glauben, das Licht,

den einst der Sommer als Krone

aus großen Blüten flicht.“ (Benn 1992: 275)

4 Zudem musste Benn die ehrenwörtliche Erklärung abgeben, dass er nicht homosexuell sei. (Benn an Oelze 12. 5. 1936). 
Dieses Gedicht entstand im Jahr 1934, jenem Jahr, das Benn, der die Machtübernahme der Nationalsozialisten entschieden und begeistert vorantrieb (vgl. seine „außerordentlich unehrenhafte Rolle bei der Gleichschaltung der [Preußischen] Akademie [der Künste]" nach Sarkowicz - Mentzer 2000: 82 sowie seine Rundfunkreden wie Der neue Staat und die Intellektuellen vom 24. 4. 19335), eine starke Desillusionierung brachte. Schon im Herbst 1933 attakierte Börries von Münchhausen Benns expressionistische Gedichte als individualistische und unsittliche Literatur. Im Mai 1934 wird er von Börries von Münchhausen nochmals angegriffen und als „reinblütiger Jude“ denunziert. Nach dem sog. Röhm-Putsch (30. 6. 1934), der Liquidierung von rund 200 SA-Spitzenfunktionären und anderen politischen Gegnern des Regimes, hat Benn schließlich für die politische Enwicklung Deutschlands nur den folgenden Satz parat: „Es giebt keine Worte mehr für diese Tragödie.“ (Benns Brief an Oelze, 24. 7. 1934, zitiert in Hillebrand 1992: 600)

Wo es keine Worte gibt, kann es Bilder geben. In diesem Zusammenhang bekommt die kleine, weiße, unscheinbare Frühlingsblume Anemone fast so etwas wie eine Signalwirkung. Sie ist weit entfernt von der „Kleine[n] Aster“ des Expressionisten Benn, der Herbstblume, die apart „dunkelhellila“ (Zeile 2) ist (Benn 1992: 21) und dem Leichnam eines „ersoffene[n] Bierfahrer[s]“ (Zeile 1) dekadent zwischen den Zähnen klemmt, bis das lyrische Subjekt sie in die Bauchhöhle des Toten auf dem Obduktionstisch mit den Worten befördert: „Ruhe sanft,/ kleine Aster!“ (Benn 1992: 21) Aus dem Bauchraum einer unbestatteten Leiche wird somit, unter reichlicher Verwendung einer Ästhetik der Abscheu, eine Vase.

Im Unterschied zu diesem kühlen und kühnen, das bürgerliche Publikum von Anfang an schockieren wollenden Gedicht, dem ersten Teil des Morgue-Zyklus Benns von 1912, wird das Anemone-Gedicht ein „Klassiker“ genannt, wozu wohl vor allem die „klassische Vierzeilerstrophe“, der „von Horaz bis Heine zu letzter Raffinesse ausgefeilte[] Quatrain“, beigetragen haben könnte, mit ihrem ausgeglichenen gekreuzten Reim sowie auch das generelle, quasi überzeitliche Thema Natur.

Aber ist das Gedicht tatsächlich apolitisch? Gleich die erste Strophe macht die stark melodisch, ja magisch klingende Blume (vor allem die vier aufeinanderfolgenden Vokale „a“-,e“-,, ,-„, e“ mit dem Akzent auf dem runden „o“ in der dritten Silbe modellieren Sinnliches und Geheimnisvolles) eigentlich zum Aufrührer. Sie erschüttert und soll erschüttern, aber dabei bleibt das Gedicht nicht stehen, mit einem ungeduldigen Komma folgt nach der dual strukturierten ersten Zeile (zwei mal vier Silben getrennt von gleich zwei Interpunktionszeichen) die Zeile zwei, die den Ist-Zustand der Welt beschreibt, den Ausgangspunkt des Gedichts: „die Erde ist kalt, ist Nichts“.

Auf dieses „Nichts“, das ein weiteres Komma von den durch ein Enjambement verbundenen Zeilen drei und vier trennt, reimt sich dabei das, was die helle Krone der

5 Nur zur Illustration seiner rauschhaften, die Weimarer Demokratie untergrabenden Rhetorik ein Zitat aus dieser Rede:

„Die Geschichte verfährt nicht demokratisch, sondern elementar. Sie läßt nicht abstimmen, sondern sie schickt den neuen biologischen Typ vor, sie hat keine andere Methode, hier ist er, nun handle und leide, baue die Idee deiner Generation und deiner Art in den Stoff der Zeit, weiche nicht, handle und leide, wie das Gesetz des Lebens es befiehlt.“ (Benn zitiert nach Zimmermann 1992: 44) 
Anemone symbolisiert, nämlich „ein Wort des Glaubens, des Lichts“. Der Kälte und der Vernichtung der Blut-und-Boden-Ideologie, könnte man schlussfolgern, wenn das Gedicht in den Koordinaten der damaligen Zeit gelesen wird, antwortet das subversiv und gleichsam im Traum Gemurmelte und Unschuldige der Blume, das schließlich hoffnungsvoll aufleuchtet.

Dass dem Ausdruck „Erde“ tatsächlich diese ideologische Färbung zukommt, beweist die zentrale zweite Strophe. Hier wird dem Dunkel, das gewöhnlich das Symbol der Erde prägt (vgl. Becker 1992: 73), die eindeutig negative, ethisch konnotierte Charakterisierung „ohne Güte“ beigegeben, eine Personifizierung, die in der nächsten Zeile noch ausgebaut wird zu der Vorstellung, dass dieser Erde „nur die Macht gerät.“ (Zeile 6) Und diese Macht ist zugleich auch laut (wie die vielen Reden, Kundgebungen und Märsche der NS-Zeit), wohingegen die Blüten der Anemone „leise“ sind und „so schweigend hingesät."

Wie eine Beschwörung wird dann in der abschließenden dritten Strophe die Blume Anemone nochmals aufgerufen und mit den zuversichtlichen Zeilen versehen, dass sie „den Glauben, das Licht“ für den „Sommer als Krone“ trage, allerdings verlegt in das unbestimmte und in eine ferne Zukunft verweisende Adverb „einst“. Diese Strophe kopiert zwar den Bau der Strophe 1, rekapituliert die pathetische Ansprache der Blume, positioniert auch das Enjambement symmetrisch zu dem der ersten Strophe (Zeilen 3 und 4 sowie 11 und 12). Aber zugleich ist sie das Finale des ganzen Gedichts, dessen Vision des Sommers ebenso unscheinbar und bescheiden, in fast von selbst fließenden Reimen präsentiert wird wie die kleinen Blüten der Blume als mutige Vorkämpfer des Frühlings. Somit stellt dieses leise Gedicht ein prägnantes und frühes Beispiel für Benns ,innere Emigration' dar, die mit ganz anderen, ausgefeilten, höchst kultivierten, doch zugleich originellen und keineswegs traditionalistischen Waffen zu Felde zog als der Autor selbst zur Zeit seiner einjährigen nationalsozialistischen Verblendung.

Doch das Jahr 1936 lässt Benns Geschichtspessimismus angesichts der politischen Verhältnisse überwiegen, nicht zuletzt durch die propagandistisch ausgelotete Olympiade in Berlin vom 1. bis zum 16. August 1936. Wieder in drei vierzeiligen Strophen wie im Anemone-Gedicht, also klassisch, formuliert der Autor nun im Text Einsamer nie - , der nach 1945 zum „Kanongedicht der Gegenwartslyrik“ wurde (Korte 1999: 85), seine diametral veränderte Wahrnehmung der zeitgenössischen Wirklichkeit wie der Rolle des (dichtenden) Individuums darin. Erst hier wird dem lyrischen Subjekt in der Du-Ansprache ein Bezirk des „Gegenglück[s]“, des „Geist[es]“, klar zugewiesen als dem Ort einer gewissermaßen stummen Revolte, der trotzdem gedient werden muss, die erst mit Mühe $\mathrm{zu}$ verwirklichen ist:

„Einsamer nie -

Einsamer nie als im August:

Erfüllungsstunde -, im Gelände

die roten und die goldenen Brände,

doch wo ist deiner Gärten Lust? 


\begin{abstract}
Die Seen hell, die Himmel weich, die Äcker rein und glänzen leise, doch wo sind Sieg und Siegsbeweise aus dem von dir vertretenen Reich?
\end{abstract}

Wo alles sich durch Glück beweist und tauscht den Blick und tauscht die Ringe im Weingeruch, im Rausch der Dinge, -: dienst du dem Gegenglück, dem Geist.“ (Benn 1992: 281)

Dies ist ein zutiefst melancholisches Gedicht, dessen „August“ als Höhepunkt des Sommers, jenes Sommers, dessen Kommen ja das Anemone-Gedicht zwei Jahre zuvor so hoffnungsvoll ankündigte, sich nun mit dem Höhepunkt der nationalsozialistischen Propagandamaschinerie im Rahmen der Berliner Olympiade decken soll (mit Realien wie dem Aufmarschgelände mit ,Führertribüne‘), doch für das lyrische Subjekt sich nicht decken kann. Die Erfüllungsstunde der Umgebung ist seine Erfüllungsstunde nicht.

Auch die „roten und goldenen Brände“, die dieser Umgebung zugewiesen werden und nach Theo Buck dem roten Mohn und dem goldenen Weizen (Buck 2006) entsprechen, allerdings auch mit dem ersten olympischen Fackellauf in der Geschichte der olympischen Spiele während der Eröffnungsfeier zusammenhängen könnten, sind dem lyrischen Ich fremd, es registriert sie, sehnt sich aber nach weniger spektakulären, dafür eigenen Gärten, die jedoch unauffindbar bleiben.

Das (nationalsozialistische) Reich präsentiert sich indessen, wie es die beiden ersten Zeilen der zweiten Strophe ausführen, mit „hell[en]“ Seen, „weich[en]“ Himmeln und „rein[en]“ und zudem verhalten „glänzen[den]“ Äckern, also idyllisch, was alles Beweise des Siegs seiner Ideologie erbringt. Das lyrische Ich kann sich an ihr aber nicht beteiligen und sucht nach einem eigenen, alternativen Reich, bestimmt auch in politischer Hinsicht.

Noch in der letzten Strophe distanziert sich das lyrische Ich von seiner Umgebung, deren Skizzierung diesmal sogar volle drei Zeilen gewidmet werden. Sie zeichnet sich durch bewiesenes, offenbar plakatives Glück aus, durch den Tausch der Blicke als einem Wegsehen von allen unerwünschten Realitäten und Problemen sowie durch den Tausch der Ringe, d. h. der Treue (z. B. zu kulturellen Traditionen) und Zugehörigkeiten (vgl. symbolische Bedeutung von Ring in Becker 1992: 242-243), wobei diese beiden Tausche (die auch täuschen sollen) zudem „im Weingeruch, im Rausch der Dinge“ erfolgen, was ein klarer Hinweis auf den Fanatismus des ,Dritten Reichs“ ist, den ja Benn auch eine Zeit lang teilte.

Angesichts all dessen stockt zunächst der lyrische Fluss, und gab es im ähnlich gebauten Anemone-Gedicht eine bei Benn öfters anzutreffende graphische ,Kollision“ von Gedankenstrich und Doppelpunkt („Erschütterer -: Anemone“), kommt es kurz vor dem Finale des Gedichts Einsamer nie - zum Gleichlauf sogar dreier Interpunktionszeichen: des Kommas, des Gedankenstrichs und des Doppelpunkts („im Weingeruch, im Rausch der Dinge, -:“). 
Nach dieser graphisch herausgehobenen Coda erst, die der äußeren, politischen Wirklichkeit zu allem Möglichen noch Türen offen lässt, definiert das Du seine Aufgabe angesichts der Zeitumstände wie der Berliner Olympiade: es soll still dem „Gegenglück“ dienen, „dem Geist“. Mit dieser Rhetorik etabliert sich Benn als Autor der ,Inneren Emigration“ und als solcher will er dann vor allem nach dem Ende des Zweiten Weltkriegs gesehen werden. Das bürgerliche Publikum der restaurativen Adenauer-Ära erfüllt ihm diesen Traum und katapultiert Benn zum Stardichter der bundesdeutschen 50er Jahre. (vgl. Korte 1999: 85-86)

Ähnlich populär wurde in der BRD der 1950er Jahre auch der eine Generation jüngere Günter Eich, auch er ein Autor, der „lange vor 1945 zu schreiben und zu publizieren“ begann (Korte 1999: 86). Nach 1945 wurde Eich jedoch schlagartig und auf eine ganz andere Art als Gottfried Benn bekannt, nämlich, indem er mit seinen formal wie inhaltlich innovativen Gedichten Latrine und Inventur so etwas wie Grundtexte der sog. Kahlschlagliteratur schuf. Beide Texte werden als Kriegsgefangenschaftsgedichte angesehen, die - wie sehr sie im Rahmen der Eichschen Lyrikproduktion dieser Zeit eine Ausnahme bilden $^{6}$ - der veränderten gesellschaftlichen Landschaft Deutschlands in eigenständigen, abgehärteten Versen Rechnung tragen. Der ältere Text Latrine verwendet noch Reime, wenn auch nur abwechselnd mit dem Vers Libre, und schockiert durch seine Thematik und die reichliche Verwendung einer Ästhetik der Abscheu, deren Einsatz das Gedicht in die Nähe des erwähnten expressionistischen Morgue-Gedichts Kleine Aster von Gottfried Benn stellt:

„Latrine

Über stinkendem Graben, Papier voll Blut und Urin, umschwirrt von funkelnden Fliegen, hocke ich in den Knien, den Blick auf bewaldete Ufer, Gärten, gestrandetes Boot. In den Schlamm der Verwesung klatscht der versteinte Kot. Irr mir im Ohre schallen Verse von Hölderlin.

In schneeiger Reinheit spiegeln

Wolken sich im Urin.

Geh aber nun und grüße

die schöne Garonne

Unter den schwankenden Füßen

schwimmen die Wolken davon.“ (Eich 1989: 9)

$6 \quad$ Zu dieser Zeit und bis zum Anfang der 50er Jahre schreibt Eich noch in der Tradition der naturmagischen Lyrik. (vgl. Lamping 2008: 39-42) 
Doch wenn sich in diesem Gedicht der von den Nationalsozialisten häufig eingesetzte und missbrauchte Klassiker Hölderlin ausgerechnet auf „Urin“ reimt (als Teil des quasi ,klassischen' Quatrains zudem, dessen Reim nur, wie erwähnt, ein wenig ,hinkt'), so bedeutet es wohl mehr als nur das Bennsche Provozieren-Wollen des satten Bildungsbourgeois. Eich geht es hier offensichtlich darum, eine möglichst große, ja äußerste Distanz zu den schönen überlieferten Worten wie Hölderlins „Was bleibet aber, stiften die Dichter" vom Ende seiner Hymne Andenken zu schaffen. Indem Eich Abschnitte aus dieser Hymne zitiert (Zeile 13 und 14: „Geh aber nun und grüße/ die schöne Garonne“), dem Zitat aber die rauhe Realität des Krieges oder Kriegsgefangenenlagers (Strophen 1 und 2: „stinkende[r] Graben“, „Blut und Urin“, „Schlamm der Verwesung“, „der versteinte Kot“) sowie ihre symbolische Überhöhung (,gestrandetes Boot“) entgegensetzt, will er ein anderes, ungeschminktes, vorurteilsloses Andenken an diese Realität provozieren und festhalten. Fünf Jahre später, 1950, wird er noch weiter gehen und in seinem Gedicht Träume direkt fordern:

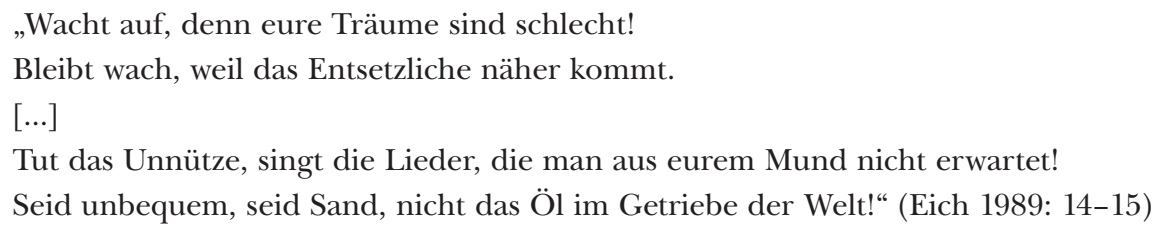

Die Diskrepanz zwischen den Werten und Hochleistungen der deutschen Geisteskultur einerseits und den fatalen Zerstörungen, dem allgemeinen Ruin infolge des Krieges andererseits thematisiert auch die zur gleichen Generation wie Eich gehörende Marie Luise Kaschnitz, wenn auch viel ,gepflegter', traditioneller; so ist, im Unterschied zu Eich, ihr gekreuzter Reim noch völlig intakt. Im zeitgemäßen Genre der Trümmerlyrik, dem Wilfried Barner neben dem des Heimkehrgedichts „durchaus wichtige und weiterführende Impulse, sich der Last des jüngst Erfahrenen [...] zu stellen“ (Barner 1994: 76), einräumt, beschreibt sie in ihrem nüchtern benannten Gedichtzyklus Rückkehr nach Frankfurt, gleich in der ersten Nummer der Zeitschrift Die Wandlung 1945/46 publiziert, ihre Wiederbegegnung mit der Goethe-Stadt nach deren Bombardierungen. Dabei konfrontiert sie die Trümmer des Geburtshauses von Goethe mit dem absurden Schild, wonach sie „Besitz der Nation“ seien, und schließlich mit dem Geist des Klassikers selbst:

\footnotetext{
„Und das Haus war ein Loch, ein Kellerschacht,

Ein Haufen Dreck zum Hohn,

Und Schilder waren dort angebracht,

Darauf stand: Besitz der Nation.

Ich las die Zeichen traumgenau

Über dem wehenden Gras,

Gestalten bückten sich ins Grau

Und sammelten irgend etwas.

Und plötzlich stand am Straßenrand
} 
Er selber in Fleisch und Blut,

Er trug nicht den blauen Rock mit dem Band

Und nicht den Campagnahut.

Er trug nicht einmal sein eignes Gesicht,

Ich wußte nur: er war da.

Und ich erschrak wie vorm Jüngsten Gericht,

Weil er sein Haus ansah.

[...]“ (Kaschnitz 1985: 150)

Hier stellt noch Goethe eine unanzweifelbare, das Ich mit fast religiösem Schauder erfüllende Größe dar, die mit dem „Haufen Dreck“, mit dem „wehenden Gras“ der Ruinen und den sie durchsuchenden Menschen schrill kontrastiert. Erst diese imaginierte Begegnung mit dem Klassiker zeigt dem lyrischen Ich und mit ihm den Deutschen, wie tief ihre Kultur gesunken ist, und weist auf das Ausmaß der Zerstörungen nicht nur im Materiellen, sondern auch im Geistigen hin.

Dies sollte aber bald grundsätzlich überboten werden, und zwar von dem eine Generation jüngeren jüdischen Autor Paul Celan aus Czernowitz in der Bukowina. Sein berühmtes Gedicht Todesfuge geht viel radikaler mit deutscher Tradition und den Autoritäten und Werten der deutschsprachigen Kultur um, radikaler, ernster, verbindlicher auch als Eich, und lässt sie angesichts der Shoah völlig außer Kraft treten.

Zudem ist dieser Text einer der ersten und markantesten, die auf den Völkermord an den europäischen Juden eingehen, was in der ersten Nachkriegszeit bis 1950 und auch noch darüber hinaus allein den jüdischen Autoren wie eben Paul Celan oder Nelly Sachs oblag. (vgl. Lamping 2008: 37) Bezeichnend sollte auch sein, dass er wie auch sein Autor häufig missverstanden wurden.

Berühmt berüchtig ist Celans ,Fiasko' bei der Tagung der Gruppe 47 in Niendorf an der Ostsee im Mai 1952, als sein Vortrag der Todesfuge, am altösterreichischen Bühnenton orientiert (vgl. Böttiger 2017: 171), nicht nur lächerlich pathetisch, sondern in einer eigenartigen Projektion ,ja wie [jener von] Goebbels“ empfunden wurde, und zwar ausgerechnet von dem durch Hitlerjugend und NS-Studentenbund sozialisierten Walter Jens. ${ }^{7}$ Sogar der Gruppenchef Hans Werner Richter spricht wenig geschmackvoll vom „Singsang [...] wie in einer Synagoge“ (zitiert in Emmerich 2001: 92). ${ }^{8}$ Eine Auseinandersetzung inhaltlicher Art wird nicht dokumentiert.

Subtiler geht Hans Egon Holthusen 1954 in seiner Merkur-Rezension Fünf junge Lyriker vor, als er nach Autoren wie Albert Arnold Scholl oder George Forestier ganz zuletzt

7 Mit fast naivem Enthusiasmus erzählt Jens über Celan: „Er wurde ausgelacht [...] Die ,Todesfuge‘ war ja ein Reinfall in der Gruppe! Das war eine völlig andere Welt, da kamen die Neorealisten nicht mit, die sozusagen mit diesem Programm großgeworden waren." (zitiert in Emmerich 2001: 92)

8 Richter hat sich immerhin für diesen informell geäußerten Vergleich entschuldigt, was nicht zuletzt aus seinem Tagebucheintrag aus der Zeit nach Celans Freitod hervorgeht: „Paul Celan verlangte Rechenschaft und versuchte mich in die Position eines ehemaligen Nationalsozialisten zu drängen. Ilse Aichinger und Ingeborg Bachmann weinten und baten mich unter Tränenströmen immer wieder, mich zu entschuldigen, was ich dann schließlich tat. Paul Celan hat es mir nie vergessen." (Richter 2012: 158) 
Paul Celan behandelt. Er unterstreicht gleich zu Anfang, dass dieser jüdische Autor vom „östlichen Rande des deutschen Sprachgebiets“ stamme und, was der in Paris lebende Celan antisemitisch wertet, ${ }^{9}$ „ein Fremdling und Außenseiter der dichterischen Rede“ sei. (Holthusen 1954: 385) Es folgen kritische Passagen darüber, dass Celans Sprache sich „an sich selbst entzünde[]“ (Holthusen 1954: 385) und zu sehr unter französischem Einfluss stehe (vgl. Holthusen 1954: 385), wobei ein Verweis auf die Nähe zu Iwan Goll als eine kleine Invektive im Rahmen der sog. Goll-Affäre (nach Barbara Wiedemann Phase II der ,Infamie‘: 1953-1959) gewertet werden könnte. (vgl. Holthusen 1954: 386)

Doch die Todesfuge wird gelobt: „Instinkt und Leidenschaft“ Celans haben sich hier „mit dem Kairos eines großen Motivs“ (sprich der Shoah) zu „etwas Eigenartige[m]“ verbinden können, dem Autor sei es zudem gelungen, „eines der schrecklichsten und bedeutsamsten Ereignisse der jüngsten Geschichte, den massenhaften Verbrennungstod der Juden in deutschen Konzentrationslagern, in einer Sprache zu besingen, die von der ersten bis zur letzten Zeile wahre und reine Dichtung ist, ohne eine Spur von Reportage, Propaganda und Räsonnement.“ (Holthusen 1954: 390)

Dieser letzte Zusatz (neben der Euphemisierung des Mordes an den europäischen Juden zum „Verbrennungstod“), wie positiv er sich auch gebärdet, nimmt allerdings dem Celanschen Text gerade jenen Realitätsgehalt, der bereits bei der ersten, rumänischen Publikation des Gedichts durch die Notiz des Herausgebers akzentuiert wurde: „Das Gedicht [...] beruht auf der Beschwörung einer wahren Begebenheit. In Lublin wie in anderen ,nazistischen Todeslagern' zwang man eine Gruppe von Verurteilten, wehmütige Lieder zu singen, während andere Gräber schaufelten." (Zitiert in Felstiner 2000: 56). Dass zudem das Realistische, Reportagehafte für Holthusen durchaus positiv besetzt wird und anzustreben ist, beweist die Selbstcharakterisierung eigener Gedichte in dem Aufsatz Deutsche Literatur nach dem Zweiten Weltkrieg von 1955: in den Gedichten Holthusens komme, so Holthusen, „eine ,barocke“ Spannung zwischen kraß realistischen, gelegentlich reportagehaften Elementen und weltüberwindenden Himmelfahrtsmotiven zum Ausdruck." (Holthusen 1955: 262) De facto annihiliert also Holthusen Celans ,Grabmal' für dessen in einem Vernichtungslager ermordete Mutter durch die Zuweisung der Todesfuge zur „reine[n] Dichtung“ (Holthusen 1954: 390); und zwar wohl wissend um die symbolische Bedeutsamkeit des Textes für die Privatperson des Autors.

Wenn Holthusen zum Motiv des Trinkens in der „Todesfuge“ außerdem ausführt: „Trinken ist Sterben als äußerste Selbstverwirklichung, Opfertod, Aufsteigen zu einem „Grab in der Luft" (Holthusen 1954: 390), fröstelt es einen, denn in der Shoah eine „äußerste Selbstverwirklichung“ der Juden zu sehen, ihren „Opfertod“, setzt eine Freiwilligkeit voraus, die sie während des Genozids wahrhaftig nicht hatten. Auch Celans Bewältigung des Themas, das als „alle menschliche Fassung sprengendes, alle Grenzen der künstlerischen Einbildungskraft überschreitendes" (Holthusen 1954: 390) emphatisch hervorgehoben wird, wird mit Lob bedacht, einem zwiespältigen jedoch. Durch diese handwerkliche Bewältigung könne man nämlich „der blutigen Schreckenskammer der Geschichte entfliegen [...], um aufzusteigen in den Äther der reinen Poesie.“ (Holthusen

9 Von der SS-Vergangenheit Holthusens wusste Celan. (vgl. Wiedemann 2000: 208) 
1954: 390) Das Schlimmste wird damit harmonisiert und die Todesfuge als „eines der großartigsten Zeitgedichte, [...] die wir besitzen“, dem Wir der Etablierten einverleibt, über welches die Koryphäe des westdeutschen Literaturbetriebs Holthusen frei verfügt. ,Der arme Poet ' Celan dagegen, „[e]in einsamer, dem aktuellen Kräftespiel der heutigen deutschen Lyrik scheinbar ganz abgekehrter Dichter“ (Holthusen 1954: 390), wird in seinem Anliegen, die Shoah in ihrer alle Maße sprengenden Singularität artikulierbar und somit auch den Nicht-Betroffenen nachvollziehbar zu machen, marginalisiert.

\section{Fazit}

Mit der bedingungslosen Kapitulation des sogenannten Deutschen Reichs endete am 8. Mai 1945 die nationalsozialistische Terrorherrschaft wie der schlimmste und grausamste Krieg der gesamten Menschheitsgeschichte, der weltweit rund 55 Millionen Tote kostete. Die totale militärische Niederlage brachte wirtschaftlichen Ruin, den Zusammenbruch der Infrastruktur, Hunger und Flüchtlingsströme mit sich, es herrschte die anfangs erwähnte „nachsintflutliche Atmosphäre“ (Žmegač 1981: 374), allerdings auch eine „Kultureuphorie“ (Glaser 1990: 17).

Beides führte dazu, dass man im Hinblick auf Literatur und Kultur von der „Stunde Null“ oder „Stunde X“ (Forster - Riegel 1995: 360) sprach und schrieb, vom „Nullpunkt“, den es zu überwinden galt (Holthusen 1952: 137), vom „Kahlschlag in unserem Dickicht“, der einen radikalen Neubeginn nach 1945 herbeiführen sollte (Wolfgang Weyhrauch im Vorwort zur Anthologie Tausend Gramm, 1949), vom „absoluten Indifferenzpunkt“ der „Tabula rasa“ (beides Holthusen; vgl. Korte 2004: 9), von der ,Trümmerliteratur", aber auch von ,Bestandsaufname“ und ,Inventur'.

All diese Begriffe legten nahe, dass der 8. Mai 1945 einen sehr markanten Bruch in der deutschsprachigen Kultur bedeutete, vom Pathos des Neubeginns getragen. Bald sollte sich jedoch zeigen, dass mit ihm auch Tabus, Manipulationen und Verschleierungen einhergingen. Die Kontinuitäten zwischen dem ,Tausendjährigen Reich` und der Gegenwart, aber auch jene zwischen der Vorkriegszeit und der Gegenwart, wurden ignoriert, man meinte in einer Tabula-rasa-Situation zu leben, wo alles neu entwickelt wird (die Schlüsselbegriffe waren „der kulturelle Aufbau und Aufstieg, die kulturelle Wandlung und Besinnung“ (Glaser 1990: 9)), und nichts Altes mehr gilt.

Allerdings war beispielsweise die „Tabula-rasa“-Metapher, neben jener der „Überwindung des Nullpunkts“ - beiden sprach Hans Egon Holthusen das Wort -, bezeichnenderweise an ein Gedicht Holthusens gebunden, das schon 1943 vorlag, also die Kontinuität eher bezeugte als den herbeigesehnten, da entlastenden Traditionsbruch. Dass sich dieses sogleich „Tabula rasa“ benannte Gedicht affirmativ auf den Zweiten Weltkrieg bezieht, jenen Krieg, den der Autor zwei Jahre zuvor im Essay Deutscher Geist im Kriege noch eifrig rechtfertigte, beweist Robert Rduch durch eine Motivanalyse des Textes. ${ }^{10}$ (vgl. Rduch 2012: 154-155)

10 Ein Jahr nach der Entstehung des Gedichts soll sich zudem Holthusen, wie schon erwähnt, noch in SS-Uniform gezeigt haben. (vgl. Rduch 2012: 148) 
Doch Holthusen bot das Gedicht gleich der ersten Nummer der in amerikanischer Lizenz erscheinenden, von Karl Jaspers, Dolf Sternberger, Werner Krauss und Alfred Weber herausgegebenen Zeitschrift Die Wandlung an, die ein „neues, [den] aufklärerischen Intentionen [des Herausgebers Dolf Sternberger] entsprechendes politisch-literarisches Forum“ (Gersdorff 1992: 151-152) schaffen wollte. Es wurde tatsächlich angenommen und erschien im November 1945 im ersten Heft der Wandlung, was Holthusen Mitte der 60er Jahre veranlasste, den Text zum „lyrische[n] Zeugnis eines deutschen Sinneswandels" (zitiert in Rduch 2012: 149) zu erklären.

Aber nicht nur wegen Opportunisten wie Hans Egon Holthusen kann man in der deutschsprachigen Lyrik weder vom ,Kahlschlag“ noch von der "Stunde Null' sprechen. (vgl. Korte 2004: 5) Wie wir gesehen haben, gelingt auch bei Gottfried Benn, dem Kultlyriker der bundesdeutschen 1950er Jahre, rezeptionsgeschichtlich gesehen der Durchbruch nach dem Ende des Weltkriegs weitgehend mit Gedichten, die bereits in den 30er Jahren geschrieben und zum Teil auch damals schon veröffentlicht wurden und die im positiven Sinne traditionalistisch waren. Vor allem sein Text Einsamer nie -, den er nach der ersten Publikation in den ,gereinigten' Ausgewählten Gedichten im Dezember 1936 seinen Statischen Gedichten angliedert, die 1948 in der Schweiz und 1949 in Deutschland erschienen (Buck 2006), ist also kein für die 50er Jahre entworfenes, auf die gesellschaftliche Lage nach 1945 antwortendes Gedicht. Sogar das bekannteste Gedicht Gottfried Benns, Verlorenes Ich, das die Nachkriegspoetik des Autors so gut zu illustrieren scheint, liegt bereits 1943 vor; sein Erfolg in den 1950er Jahren zeugt ebenfalls davon, dass nach 1945 eher Kontinuitäten gefragt waren als ein völliger und ,nackter ${ }^{\star}$ Neuanfang.

Auch bei Marie Luise Kaschnitz kann man anhand ihres Gedichtzyklus Rückkehr nach Frankfurt in formaler Hinsicht eher von einer Fortsetzung als vom Bruch sprechen. Zwar ist das Thema dieser Gedichte neu und höchst aktuell (rein inhaltlich kann ihre Lyrik gleich zwei für die Zeit nach 1945 typischen Genres zugeordnet werden: der Trümmerwie der - weiblichen - Heimkehrerlyrik (vgl. Barner 1994: 76)).

Aber schon ein flüchtiger Blick auf die sprachliche Umsetzung dieser Verse zeigt, wie traditionell sie im Kern noch sind. Sie wenden zwar Ironie an, ,singen" jedoch unangezweifelt weiter mithilfe ausgeglichener, überlieferter Metren und eines sehr regelmäBigen Reims. Auch ihr Wortschatz ist trotz aller Gegenwartsnähe wenig innovativ. Die massiven Kriegszerstörungen haben die Nabelschnur zur Tradition nicht zerschnitten, schließlich ist auch das Etikett Goethe hier noch weitgehend intakt. So dass man bei Kaschnitz in Hinsicht auf das Jahr 1945 von einer Hybridität sprechen kann: die Inhalte sind zeitgenössisch und neu, engagiert, die Form noch die Tradition reflektierend, was jedoch der Aufnahme beim bürgerlichen Publikum durchaus förderlich sein durfte. Erst mit der Sammlung Neue Gedichte von 1957 befreit sich Kaschnitz von ihrer diesbezüglichen Treue.

Dagegen haben wir es bereits bei Eichs Gedicht Latrine, dessen Entstehung Axel Vieregg 1991 sogar schon auf das Jahr 1940 zurückdatiert, mit der modernen Technik der Collage zu tun und mit mutigen und provozierenden, frischen Versen. Ihr Reim ist brüchig, der ikonische Text Inventur verzichtet auf ihn ganz. Beide Texte scheinen Wolfdietrich Schnurres Forderung an das Nachkriegsgedicht zu erfüllen: „zerschlagt 
eure Lieder/ verbrennt eure Verse/ sagt nackt/ was ihr müßt.“ (Schnurre in: Schnell 2008: 495)

Dies trifft nicht zuletzt auch für das Gedicht Schuld von Albrecht Haushofer zu, obwohl die Form des Sonetts, die er für seine Aussage wählte und die er konsequent befolgte, zu den angesehensten und strengsten, auch den traditionellsten zählt. Doch inhaltlich kann hier sehr wohl ein „Sinneswandel“ (Holthusen zitiert in Rduch 2012: 149) festgestellt werden, der für jede Zäsur unerlässlich ist.

Konnte die Form bei Haushofers lyrischen Bekenntnissen noch weitgehend intakt bleiben, so erfuhr sie unter dem Schock der Shoah bei jüdischen Lyrikern wie Paul Celan oder Nelly Sachs einen sehr radikalen Bruch. Diese Gedichte, die bereits ab 1943 (Sachs) bzw. 1944 (Celan) entstehen und auf den Verlust ihrer Nächsten in den Todeslagern reagieren, sind formal wie inhaltlich innovativ, müssen jedoch auf die Gunst der Leser bis in die 50er Jahre hinein warten. Zu beschäftigt sind die Deutschen „mit ihrem eigenen Leid, um Verständnis für die Opfer aufzubringen“ (Olschner 1992: 280), so dass das Echo der Sachsschen Sammlung In den Wohnungen des Todes, bereits 1947 in der Sowjetischen Besatzungszone veröffentlicht, im Vergleich mit der Resonanz von Benns Statischen Gedichten (1948) oder Bechers Heimkehr (1946) als „recht karg“ zu bezeichnen ist. (Olschner 1992: 272).

So scheint dem Meilenstein 1945 eher eine symbolische Bedeutung innezuwohnen. Er begrenzt den Zweiten Weltkrieg und den ,Zivilisationsbruch Auschwitz‘ (Dan Diner) zeitlich, weist darauf hin, dass den Atrozitäten des ,Dritten Reichs“ ein Ende gemacht wurde bzw. werden sollte. Poetologisch und rezeptionsgeschichtlich gesehen erweisen sich jedoch eher die Jahre 1943/44 (Holthusen, Sachs, Celan) und 1947/48 (Sachs, Celan, Benn, Eich, Kaschnitz, Holthusen) als Zäsur. Und sie ist auch bei jedem der hier behandelten Dichter unterschiedlich gewichtet, was das Verhältnis von Alt und Neu, von Bruch und Kontinuität betrifft. Diese Mannigfaltigkeit versuchte der Beitrag in der gebotenen Kürze zu skizzieren.

\section{Primärliteratur}

Améry, Jean (2005): Brief an Hans Egon Holthusen (1967). In: ders.: Werke. Bd. 7: Aufsätze zur Politik und Zeitgeschichte. Hrsg. v. Stephan Steiner - Heidelberger-Leonard, Irene. Stuttgart: Klett-Cotta, S. 47-52.

Améry, Jean (1966): Jenseits von Schuld und Sühne. Bewältigungsversuche eines Überwältigten. München: Szczesny.

Benn, Gottfried (1992): Gesammelte Werke in der Fassung der Erstdrucke. Bd. 1: Gedichte in der Fassung der Erstdrucke. Mit einer Einführung hrsg. von Bruno Hillebrand. Frankfurt am Main: Fischer Taschenbuch.

Celan, Paul (2004): Werke. Tübinger Ausgabe. 9 Bände. Hrsg. v. Jürgen Wertheimer. Bd. 1: Mohn und Gedächtnis. Frankfurt am Main: Suhrkamp.

Eich, Günter (1989): Gedichte. Frankfurt am Main: Suhrkamp.

Haushofer, Albrecht (1979): Schuld. In: Kircher, Hartmut (hrsg.): Deutsche Sonette. Stuttgart: Philipp Reclam jun., S. 359. 
Holthusen, Hans Egon (1979): „Es war mein Blut, das sich aus dir ergossen“. In: Deutsche Sonette. Hrsg.v. Hartmut Kircher. Stuttgart: Philipp Reclam jun., S. 358.

Holthusen, Hans Egon (1966b): Freiwillig zur SS II. In: Merkur. Deutsche Zeitschrift für europäisches Denken. XX. Jahrgang, 11, S. 1037-1049.

Holthusen, Hans Egon (1966a): Freiwillig zur SS I. In: Merkur. Deutsche Zeitschrift für europäisches Denken. XX. Jahrgang,10, S. 921-939.

Holthusen, Hans Egon (1957): Tabula rasa. In: Best, Otto F. (hrsg.): Deutsche Lyrik und Prosa nach 1945. Berlin - Frankfurt am Main: S. Fischer, S. 7.

Holthusen, Hans Egon (1947): Klage um den Bruder. In: Das Gedicht. Blätter für die Dichtung. Hamburg: Heinrich Ellermann, S. 1-15.

Holthusen, Hans Egon (1940): Der Aufbruch. Aufzeichnungen aus dem polnischen Kriege. In: Eckart. Blätter für evangelische Geisteskultur 16, S. 304-307.

Hölderlin, Friedrich (1984): Gedichte. Hrsg. v. Jochen Schmidt. Frankfurt am Main: Insel Taschenbuch.

Kafka, Franz (1998): Briefe 1902-1924. Frankfurt am Main: Fischer.

Kaschnitz, Marie Luise (1985): Gesammelte Werke, Bd. V: Die Gedichte. Hrsg. v. Christian Büttrich - Norbert Miller. Frankfurt am Main: Insel.

Richter, Hans Werner (2012): Mittendrin. Die Tagebücher 1966-1972. München: Beck.

\section{Sekundärliteratur}

Arndt, Erwin (1989): Deutsche Verslehre. Bindlach: Gondrom Verlag.

Barner, Wilfried (1994): Geschichte der deutschen Literatur von 1945 bis zur Gegenwart. München: Beck.

Becker, Udo (1992): Lexikon der Symbole. Freiburg - Basel - Wien: Herder.

Buck, Theo (2006): Zu Gottfried Benns Gedicht „Einsamer nie - “. In: Text + Kritik, 44. http:// www.planetlyrik.de/theo-buck-zu-gottfried-benns-gedicht-einsamer-nie/2012/09/ (6. 10. 2017).

Böttiger, Helmut (2017): Wir sagen uns Dunkles. Die Liebesgeschichte zwischen Ingeborg Bachmann und Paul Celan. München: Deutsche Verlags-Anstalt.

Emmerich, Wolfgang (2001): Paul Celan. Reinbek bei Hamburg: Rowohlt Taschenbuch.

Felstiner, John (2000): Paul Celan. Eine Biographie. Ins Deutsche übersetzt v. Holger Fliessbach. München: Beck.

Forster, Heinz - Riegel, Paul (1995): Deutsche Literaturgeschichte, Bd. 11: Nachkriegszeit 1945-1968. München: Deutscher Taschenbuch Verlag.

Gersdorff, Dagmar von (1992): Marie Luise Kaschnitz. Eine Biographie. Frankfurt am Main Leipzig: Insel.

Glaser, Hermann (1990): Die Kulturgeschichte der Bundesrepublik Deutschland. Bd. 1. Frankfurt am Main: Fischer Taschenbuch.

Hillebrand, Bruno (1992): Biographie. In: ders. (hrsg.): Gottfried Benn: Gesammelte Werke in der Fassung der Erstdrucke. Bd. 1: Gedichte in der Fassung der Erstdrucke. Frankfurt am Main: Fischer Taschenbuch, S. 593-615.

Holthusen, Hans Egon (1955): Deutsche Literatur nach dem Zweiten Weltkrieg: Lyrik. In: ders.: Der unbehauste Mensch. Motive und Probleme der modernen Literatur. Dritte, erweiterte, neu-bearbeitete Auflage. München: Piper, S. 247-263.

Holthusen, Hans Egon (1954): Fünf junge Lyriker II. III Paul Celan. In: Merkur. Deutsche Zeitschrift für europäisches Denken. VIII. Jahrgang, Heft 6 (Mai), S. 385-390. 
Holthusen, Hans Egon (1952): Die Überwindung des Nullpunkts. Aspekte der deutschen Literatur seit 1945. In: ders.: Der unbehauste Mensch. Motive und Probleme der modernen Literatur. München: Piper, S. 137-168.

Kircher, Hartmut (1979): Hans Egon Holthusen. In: ders. (hrsg.): Deutsche Sonette. Stuttgart: Philipp Reclam jun., S. 490-491.

Korte, Hermann (2004): Deutschsprachige Lyrik seit 1945. 2., völlig neu bearbeitete Auflage. Stuttgart - Weimar: Metzler.

Korte, Hermann (1999): Energie der Brüche. Ein diachroner Blick auf die Lyrik des 20. Jahrhunderts und ihre Zäsuren. In: Text + Kritik, Sonderband XI/99, S. 63-106.

Lamping, Dieter (2008): „Wir leben in einer politischen Welt“. Lyrik und Politik seit 1945. Göttingen: Vandenhoeck \& Ruprecht.

Olscher, Leonard (1992): Der mühsame Weg von Nelly Sachs' Poesie ins literarische Bewußtsein. In: Sevin, Dieter (hrsg.): Die Resonanz des Exils. Gelungene und mißlungene Rezeption deutschsprachiger Exilautoren. Amsterdam - Atlanta: Rodopi, S. 267-285.

Rduch, Robert (2012): Korrektur der Kriegsbilder in der Lyrik von Hans Egon Holthusen. In: Egyptien, Jürgen (hrsg.): Erinnerung in Text und Bild. Zur Darstellbarkeit von Krieg und Holocaust im literarischen und filmischen Schaffen in Deutschland und Polen. Berlin: Akademie Verlag, S. 147-157.

Sarkowicz, Hans - Mentzer, Alf (2000): Literatur in Nazi-Deutschland. Ein biografisches Lexikon. Hamburg - Wien: Europa Verlag.

Schnell, Ralf (2008): Deutsche Literatur nach 1945. In: Deutsche Literaturgeschichte. Von den Anfängen bis zur Gegenwart. Von Wolfgang Beutin, Klaus Ehlert, Wolfgang Emmerich [et al.]. 7., erweiterte Auflage. Stuttgart - Weimar: J.B. Metzler, S. 427-457.

Wiedemann, Barbara (2000): Paul Celan - Die Goll-Affäre. Dokumente zu einer ,Infamie‘. Frankfurt am Main: Suhrkamp.

Zimmermann, Hans Dieter (1992): Die Ästhetisierung der Politik: Gottfried Benn. In: ders. (hrsg.): Der Wahnsinn des Jahrhunderts. Die Verantwortung der Schriftsteller in der Politik. Stuttgart: Kohlhammer, S. 43-51.

Ziolkowski, Theodore (1972): Form als Protest. Das Sonett in der Literatur des Exils und der Inneren Emigration. In: Grimm, Reinhold - Hermand, Jost (hrsg.): Exil und Innere Emigration. Third Wisconsin Workshop. Frankfurt am Main: Athenäum, S. 153-172.

Žmegač, Viktor (1981): Literatur der Gegenwart. In: ders. [et al.]: Scriptors Geschichte der deutschen Literatur. Von den Anfängen bis zur Gegenwart. Königstein/Ts.: Scriptor, S. 347-390.

Mgr. Jana Hrdličková, Ph.D. / jhrdlickova@yahoo.de

Univerzita Jana Evangelisty Purkyně v Ústí nad Labem, Filozofická fakulta, Katedra germanistiky, Pasteurova 3571/13, 40096 Ústí nad Labem, CZ 
